\title{
Cardiovascular science: opportunities for translating research into improved care
}

\author{
Eugene Braunwald
}

TIMI Study Group, Cardiovascular Division, Department of Medicine, Brigham and Women's Hospital, Department of Medicine, Harvard Medical School, Boston, Massachusetts, USA.

\begin{abstract}
Cardiovascular research is progressing on many fronts, as highlighted in the collection of Reviews in this issue of the JCI. MicroRNAs that regulate cardiac function have been implicated in cardiac disorders, and efforts to develop therapeutic antagomirs are underway. The genetic bases of several cardiac disorders, including cardiomyopathies that cause heart failure and channelopathies that underlie cardiac arrhythmias, have been elucidated. Genetic testing can identify asymptomatic individuals at risk, potentially leading to effective preventative measures. Growing evidence supports the role of chronic inflammation in atherosclerosis, providing new opportunities for therapeutic intervention. For heart failure, recent work suggests that cardiac regeneration using stem/progenitor cells, gene transfer, new drugs that restore normal $\mathrm{Ca}^{2+}$ cycling, and agents that reduce reperfusion injury following myocardial infarction are all viable new approaches to managing disease. Cumulatively, it seems likely that the clinical advances emerging from ongoing research will, in the foreseeable future, reduce the number of deaths in the industrialized world from cardiovascular disease.
\end{abstract}

\section{Introduction}

The striking improvements in cardiovascular care that have occurred during my six decades of professional work represent one of the most exciting chapters in the history of modern medicine. Research in a broad array of fundamental, translational, clinical, and epidemiologic disciplines has provided the basis for the enormous strides in the prevention, diagnosis, and treatment of cardiovascular disorders $(1,2)$.

Taking a broad view of the field, cardiovascular science and care today could be considered to be a glass that is half full. Despite the above-mentioned advances, cardiovascular disease remains the most common cause of death in industrialized nations and its prevalence is rising rapidly in developing nations. This problem is due in part to our lack of full understanding of the pathobiology of these conditions, and in part to failure to fully apply the information that has already been established. Fortunately, however, cardiovascular research remains strong on many fronts. The goal of this collection of Reviews on cardiovascular research is to provide an update on some of the exciting recent developments in this field. It is not meant to be exhaustive or even close to complete, but instead to focus on a few of the interesting areas that may soon be translated into improved clinical care.

\section{MicroRNAs}

One of the most exciting chapters in modern biology opened in 1993 with the publication of two back-to-back papers in Cell by Ambros and Ruvkun and their colleagues $(3,4)$. Using C. elegans as a model, they discovered a new and unexpected regulatory mechanism involving small, non-protein-coding transcripts of RNA, subsequently named microRNAs (miRNAs). Other miRNAs followed, and it soon became apparent that these molecules are widely conserved across all forms of life. The first group of diseases studied was cancer, in which some miRNAs with altered expressions appear to act as tumor stimulants and others as tumor sup-

Conflict of interest: The author has declared that no conflict of interest exists. Citation for this article: J Clin Invest. 2013;123(1):6-10. doi:10.1172/JCI67541. pressors. In 2005 it was discovered that miRNAs can be antagonized with antisense oligonucleotides (anti-miRNAs, now referred to as antagomirs) (5). In the same year, Olson and his collaborators isolated an miRNA that influences cardiac proliferation (6).

More than 1,000 miRNAs have now been isolated, and new miRNAs continue to emerge; intense research efforts are focused on probing their multiple functions. miRNAs may enter the circulation and may be involved in communication between distant cells; some are becoming clinically useful biomarkers. Antagomirs are under development with the goal of creating new drug candidates. The Review by Quiat and Olson (7) provides an introduction to the cardiovascular implications of this important field of biology.

\section{Dilated cardiomyopathy}

In 1957, Wallace Brigden, a well-known London cardiologist, described a group of patients with "uncommon myocardial diseases: the non-coronary cardiomyopathies" (8). He pointed out that many of these are familial disorders of cardiac muscle. When I heard him deliver an early lecture on this subject, Brigden did not yet appreciate that he had identified, and was in the process of defining, a frequent cause of death or heart failure. One of the most common of the cardiomyopathies that he described is characterized by cardiac dilatation and congestive heart failure, and is now referred to as dilated cardiomyopathy (DC).

Although toxic (most commonly alcoholic) and infectious (especially viral) causes of DC were recognized in the 1970s, in 1981 a familial association was identified (9). Depending on the screening method employed, the familial form is present in between $25 \%$ and $65 \%$ of patients with DC (10). TTN, the gene encoding the sarcomeric protein titin, exhibited truncating mutations in $25 \%$ of cases of familial DC (11). From the Review by McNally, Golbus, and Puckelwartz (12), we learn that at least 50 different single genes that are linked to DC have been identified, and that their number is growing. Transmission is most commonly autosomal dominant, with variable expressivity; mitochondrial mutation with maternal transmission has also been described. Genetic analysis, employing parallel sequencing technologies, 
may enhance prognostication. Not surprisingly, the prognosis is also affected importantly by cardiovascular comorbidities such as hypertensive, ischemic, and valvular heart disease.

Genetic assessment of family members of patients with familial DC can identify asymptomatic carriers who should receive genetic counseling and be followed closely, and perhaps be treated with $\beta$-blockers and/or blockers of the renin-angiotensin-aldosterone system to prevent progression. Clinical trials of such measures would be very desirable as an increasing number of asymptomatic carriers are identified.

\section{Adaptive immunity in atherogenesis}

Atherosclerosis, the most common cause of coronary, cerebrovascular, and peripheral arterial diseases, occurs in epidemic proportions today. Accordingly, the pathogenesis of this disorder has received considerable attention. There is evidence that elevated levels of oxidized LDL cholesterol (oxLDL-C) and other atherosclerotic risk factors, such as hypertension and cigarette smoking, as well as localized low shear stress damage the artery's endothelial cells, which express leukocyte adhesion molecules. These surface structures capture monocytes that penetrate the intima. There, the monocytes mature into macrophages that take up oxLDL-C and thereby become foam cells. The latter produce inflammatory mediators that attract smooth muscle cells into the intima, where they proliferate and promote the accumulation of extracellular matrix. Together with the foam cells, the extracellular matrix contributes to the growth of atherosclerotic plaques.

Maladaptive immune responses that enhance inflammation of the arterial wall play an important role in the pathogenesis of atherosclerosis (13). Considerable research is underway in an effort to suppress these responses. However, the translation of these concepts into clinical care is challenging. Corticosteroids and immunosuppressive drugs entail hazards when administered chronically. The search is ongoing for anti-inflammatory drugs that can be administered in doses that inhibit vascular inflammation while producing a tolerable reduction of immune function and other adverse effects. Two such drugs are currently under investigation in phase 3 clinical trials. One is canakinumab, a human antibody that blocks IL-1 $\beta$, a pro-inflammatory cytokine (14). The other is low-dose methotrexate (15).

Lichtman et al. provide a helpful summary of the complex interplay between hypercholesterolemia, thrombosis, and acquired immunity in the production of atherosclerosis, which is now well established as an inflammatory disorder (16).

\section{Cardiac hypertrophy and failure}

Despite the advances in the diagnosis and management of many forms of heart disease, most patients are not cured, but the progression of their disease is slowed. Ultimately, heart failure supervenes in many patients. While mortality from acute coronary syndromes, uncontrolled hypertension, and valvular heart disease is declining, paradoxically, the prevalence of heart failure is rising. Indeed, in the United States and Europe, about 20 million patients are now estimated to suffer from chronic heart failure, and $20 \%$ of these require hospital admission each year.

Two articles in this series are devoted to providing a fuller understanding of the pathophysiology of myocardial dysfunction and using this understanding to identify new therapies. The Review by van Berlo, Maillet, and Molkentin focuses on the mechanisms of cardiac remodeling and ventricular hypertrophy, which usually begins as an adaptive response to hemodynamic stress but becomes maladaptive as the stress intensifies and/or continues (17).

A number of new approaches to the treatment of heart failure are being developed. Myofilament function is depressed in some forms of experimental heart failure consequent to activation of PKC $\alpha$ (18), and treatment with ruboxistaurin, a PKC $\alpha$ inhibitor, has improved myocardial contractility in heart failure models (19). Omecantiv mecarbil, a small-molecule cardiac myosin activator, has been shown to improve left ventricular performance in patients by increasing ejection time and stroke volume (20,21). A phase $2 \mathrm{~b}$ trial in acute heart failure with this drug is now under way (22). Notably, and in contrast to inotropes dependent on activating second messenger signaling pathways, mecarbil does not alter cyclic AMP generation, the rate of left ventricular pressure development, or myocardial $\mathrm{O}_{2}$ consumption and may represent a new approach to the treatment of systolic heart failure.

The key role that $\mathrm{Ca}^{2+}$ plays in cardiac contraction has been established for more than a century. We now know that the strength of myocyte contraction is dependent on the rate and quantity of $\mathrm{Ca}^{2+}$ released by the sarcoplasmic reticulum (SR) into the cytoplasm, where this ion activates the contractile mechanism. Myocardial relaxation is closely related to the rate of the reuptake of $\mathrm{Ca}^{2+}$ by the SR during diastole. Marks reviews the substantial evidence that the impaired contractility of the failing heart is related to depletion of SR $\mathrm{Ca}^{2+}(23)$. Abnormalities in the cardiac ryanodine receptor 2 (RYR2), also known as the $\mathrm{Ca}^{2+}$ release channel, can result in $\mathrm{Ca}^{2+}$ leaks into the cytoplasm during diastole, while the downregulation of sarcoplasmic/endoplasmic reticulum $\mathrm{Ca}^{2+}$ ATPase 2a (SERCA2a) impairs the diastolic reuptake of $\mathrm{Ca}^{2+}$ from the cytoplasm into the SR. Both of these changes reduce the $\mathrm{Ca}^{2+}$ available for release from the SR following cardiac excitation in animal models and in many patients with heart failure (24).

Efforts are underway to identify pharmacologic agents that prevent $\mathrm{Ca}^{2+}$ leak via the RYR2. Derivatives of benzothiazepines may be useful in this regard. As Marks points out, some controversy exists regarding the role of hyperphosphorylation of a particular amino acid in the RYR2 receptor in the genesis of the SR $\mathrm{Ca}^{2+}$ leak (25), but there is agreement that inhibiting this leak could be very important in the treatment of heart failure.

\section{Gene therapy}

In the early 1970s, after gene isolation became possible, it was expected that the insertion of a normal gene into diseased cells would soon lead to a new era of management of a variety of diseases. After several widely publicized missteps in early attempts to apply gene therapy, clinical research in this field was largely suspended. As happens frequently, when the pendulum for a new therapy swings widely, first in one direction and then in the other, it ultimately settles in a more realistic position. This is the case with gene therapy.

In his Review, Hajjar provides a systematic analysis of the challenges in this field (26). First he discusses the vectors and makes a cogent argument for the use of adeno-associated viruses (27). He then reviews the several methods of delivering the gene-bearing virus to the failing myocardium. A number of molecular targets are considered, and as already mentioned, there is substantial evidence that abnormalities in $\mathrm{Ca}^{2+}$ cycling play a key role in the genesis of many forms of human heart failure $(23,24)$. There are two logical points of attack on this process using gene transfer: the leaky RYR2 and the decreased activity of SERCA2a, both of which 
reduce the $\mathrm{Ca}^{2+}$ pumped back into the $\mathrm{SR}$ (28). The latter can be corrected by transfer of the SERCA2a gene (29). Such therapy had salutary effects in large animal models of heart failure (30). Human phase 1 and 2 studies have provided encouraging but not definitive results (31). Additional trials with transfer of this gene are ongoing, as are studies of other genes in heart failure.

\section{Regenerative therapy}

As a medical student in the early 1950s, I was taught that beyond early infancy the heart is a post-mitotic organ with no capacity to regenerate. An obvious corollary, curious to me at the time, was that the cardiomyocytes in elderly persons must then be the same cells that they had possessed since infancy, and that cardiac hypertrophy was due entirely to enlargement of existing cells (32).

In 1960, Linzbach showed that increased cardiac work, as occurs in hypertension and aortic valve disease, can result in hyperplasia as well as hypertrophy (33). Subsequently, Anversa and his colleagues reported that turnover of myocytes occurs in normal subjects, that this turnover is accelerated after acute myocardial infarction (34) and in end-stage heart failure (35), and that apoptosis is an important cause of cell death in ischemic and dilated cardiomyopathies (36). Bergmann et al. (37), while agreeing that there is myocyte turnover in normal hearts, believe that it is slower than proposed by Anversa's group (34).

There have been debates regarding the origins of the new myocytes in human hearts; some believe that they arise from the division of differentiated adult myocytes that have reentered the cell cycle (perhaps driven to do so), while others consider them to be the progeny of a population of resident cardiac progenitor cells $(34,38)$. When such progenitor cells are isolated, cultured, and infused into hearts damaged by ischemia, they home to the injured myocardium. Autologous hematopoietic stem cells, when infused into ischemic myocardium, can also differentiate and become precursors of cardiomyocytes, vascular endothelial cells, and smooth muscle cells. In their Review, Anversa, Kajstura, Rota, and Leri summarize the relative advantages and disadvantages of various cell types for the treatment of acute myocardial infarction and heart failure (39).

Degenhardt, Singh, and Epstein summarize research on the developing heart in an effort to identify potential myocardial progenitor cells in the adult heart (40). In this respect, the epicardium has recently emerged as a zone of particular interest (41). Epicardialderived progenitor cells can be grown in tissue culture and their proliferation stimulated by thymosin $\mathrm{B} 4$. When reinjected into the heart, these cells may serve as myocardial progenitor cells (42) and become terminally differentiated cardiomyocytes that are structurally and functionally integrated into the adult ventricle $(40,43,44)$.

Thus, the field of cardiac regeneration is now quite energetic, with many investigators, from basic biologists to clinical trialists, working in this area.

\section{Sudden cardiac death}

Sudden cardiac death (SCD) currently occurs in an estimated 300,000 to 400,000 Americans each year and stubbornly continues as one of the most common causes of death, not only in the United States but in all industrialized nations. Most victims are adults with coronary artery disease and/or congestive heart failure. Myocardial scar tissue that interferes with normal conduction appears to be an important underlying anatomic substrate in many patients. When a precipitating event, such as an acute ischemic episode, is superimposed on this substrate, a ventricular tachyarrhythmia can arise and degenerate into ventricular fibrillation, which is fatal unless it can be promptly converted by external or internal countershock.

As occurs quite often, important advances in understanding the pathobiology of a complex disorder, such as SCD in adults, can come from the analysis of monogenic forms of the condition. Although it is far less frequent in children than in adults, each year thousands of children experience unexpected SCD. Genetic abnormalities in cardiac ion channels, commonly referred to as channelopathies, are responsible for the electrophysiologic changes that may cause these serious arrhythmias. George reviews these monogenic disorders and describes recent progress in the understanding of how various mutations affect the proteins that constitute the ion channels (45). The most common and most widely studied of these disorders is congenital long QT syndrome (LQTS), which occurs in 1 of every 2,500 individuals and is characterized by considerable genetic and phenotypic heterogeneity. Indeed, 13 genetic variants and hundreds of mutations at each of the three major loci have been described for this syndrome (46).

Genetic testing for channelopathies is now becoming widely available, both in research and, increasingly, in commercial laboratories. Indeed, guidelines for the application of these tests were recently published (47). Comprehensive genetic testing is recommended for patients with or suspected of having LQTS, catecholaminergic polymorphic ventricular tachycardia, Brugada syndrome, and short QT syndrome, conditions that are discussed by George (45). In addition to aiding in diagnosis, elucidation of the genetic abnormality can also be helpful in estimating prognosis and in the selection of appropriate therapy. Whenever the diagnosis of one of these monogenic channelopathies is confirmed, genetic testing in first-degree relatives is indicated.

Until recently, basic research on the pharmacologic treatment of channelopathies utilized myocyte cultures and genetically modified animal models, but neither of these has yielded much useful information. The Review by Priori and colleagues describes a relatively new approach to the search for treatments of these conditions, that is, the use of induced pluripotent stem cells (48). Takahashi and Yamanaka (49) discovered that adult somatic cells, such as dermal fibroblasts, can be reprogrammed into differentiated cells, including myocytes, which can be cultured (50). A variety of observations can be carried out on these pluripotent cardiomyocytes, including elucidation of their electrophysiologic and mechanical properties and of their ultrastructure and organization (51).

The study of the effects of known or candidate drugs on these myocytes holds exciting potential. An early example is the finding of a pharmacologic agent that, in patients with Timothy syndrome (extreme QT prolongation accompanied by webbing of the fingers and toes) (52), abolishes the molecular abnormality of the L-type $\mathrm{Ca}^{2+}$ channel that causes frequently fatal arrhythmias. Although there is considerable interest in using induced pluripotent myocytes for the development of truly personalized medicine, Priori et al. identify a number of obstacles that must be overcome before this novel method is ready for prime time (48).

\section{Myocardial reperfusion injury}

Acute myocardial infarction caused by coronary thrombosis was first described as a clinical entity early in the twentieth century (53). The history of treatment can be divided into several phases 
(54). The first phase focused on pain relief during the acute phase, followed by bed rest for several weeks to allow healing of the injured heart. Early mortality was high and occurred in approximately $30 \%$ of patients with this condition who were admitted to a hospital. Half of these deaths were secondary to ventricular fibrillation. The development of the coronary care unit in 1961 represents the second phase of treatment (55). This involved continuous electrocardiographic monitoring and the presence of staff trained in closed chest resuscitation. In well-functioning units, mortality was immediately reduced by half.

The most common remaining cause of death was left ventricular pump failure secondary to large infarctions. Animal experiments then demonstrated that infarct size was not fixed at the instant that the coronary artery became occluded, but could be modified even hours after occlusion by improving the balance between myocardial oxygen supply and demand (56). This was followed by the third era of therapy, reperfusion of infarcting myocardium (57). When implemented promptly, mortality was reduced by about half again. However, it was soon appreciated that early reperfusion was a double-edged sword, and while it certainly salvaged severely ischemic tissue, it was simultaneously responsible for accelerating the death of potentially reversibly injured myocardium (58).

The Review by Hausenloy and Yellon (59) focuses on efforts to reduce ischemia-reperfusion injury. An important clue as to how this might be accomplished came from the work of Murry et al., who preconditioned the dog heart with brief cycles of alternating ischemia and reflow prior to a sustained occlusion, a process that reduced substantially the size of the subsequent infarction (60). Surprisingly, repetitive ischemia at a distance from the subsequent occlusion was also effective and has been referred to as "remote ischemic preconditioning" (61). Perhaps even more surprising, remote conditioning is effective even if carried out after the prolonged occlusion had begun, so called "remote postconditioning" (62).

Hausenloy and Yellon review the potential mechanism of myocardial conditioning (59). Evidence is growing that reperfusion injury is caused by a reperfusion-induced opening of mitochrondrial permeability transition pores, which damages the mitochondria and impairs ATP production. Several lines of evidence suggest that myocardial conditioning prevents opening of these pores. Proof-of-principle studies of remote postconditioning have been conducted in patients, and the results have been encouraging. Application of this approach may become the fourth era of treatment of myocardial infarction (63).

Simultaneously, as reviewed by Anversa et al. in this series (39), efforts are underway to improve myocardial function by means of cell therapy (perhaps this will become the fifth era of treatment). In patients with myocardial infarction who are left with severe left ventricular failure, gene therapy, as reviewed by Hajjar (26), may constitute the sixth era of treatment of myocardial infarction.

\section{S-nitrosylation}

In 1980, Furchgott and Zawadski published a four-page article in Nature (64). They described a remarkably simple experiment using inexpensive equipment that demonstrated the existence of a potent substance released by intact endothelium following stimulation by acetylcholine, a potent smooth muscle relaxant. This opened a totally new field of biology with vast implications for clinical medicine, especially cardiovascular medicine. Subsequently, it was discovered that $\mathrm{NO}$, derived from arginine, is the putative endothelial-derived relaxant factor. $\mathrm{NO}$ was named the molecule of the year by Science in 1992 (65), and Furchgott became a corecipient of the Nobel Prize in Physiology or Medicine in 1998.

Stamler and colleagues observed that the half-life of NO is quite brief and made the important observation that $S$-nitrosothiols (SNOs), which are derived from the reaction of $\mathrm{NO}$ with thiol groups in proteins, have a prolonged half-life, suggesting that these molecules may play an important role as NO carriers and regulate vasodilation (66). The importance of this system is also reflected in the observation that the production of the potent vasodilator SNO-hemoglobin is reduced in chronic hypoxia, which can cause pulmonary vasoconstriction and thereby play a role in the development of pulmonary hypertension and right ventricular failure (67).

The Review by Halder and Stamler (68) focuses on how SNOs coordinate $\mathrm{O}_{2}$ delivery to, and utilization by, the tissues and asserts that this system can function as a major regulator of cardiovascular performance. In the final analysis, cardiac failure can be considered to be an impairment of $\mathrm{O}_{2}$ delivery, which could be caused by reduced availability of SNOs. Further pharmacologic manipulation of this system to enhance $\mathrm{O}_{2}$ delivery may be possible, and indeed is quite likely.

\section{Conclusions}

Continuation of the research efforts described in these Reviews is likely to lead to further important improvements in cardiac care. Pursuit of strategies that inhibit maladaptive immune responses in the vascular wall could enhance prevention of atherosclerosis and its consequences. Building on the encouraging early applications of stem/progenitor cell therapy, gene therapy, and drugs that restore normal $\mathrm{Ca}^{2+}$ cycling in dysfunctional myocytes may ameliorate heart failure. Application of the growing understanding of the pathobiology of inherited channelopathies might be extended to patients with acquired arrhythmias that cause the most common forms of SCD, leading to approaches that will reduce the frequency of SCD. It is not farfetched to envisage that cardiovascular disease will in the foreseeable future be displaced from its position as the leading cause of death in industrialized nations.

Address correspondence to: Eugene Braunwald, TIMI Study Group, 350 Longwood Avenue, Boston, Massachusetts 02115, USA. Phone: 617.732.8989; Fax: 617.975.0955; E-mail: ebraunwald@partners.org.
1. Braunwald E. Shattuck lecture - cardiovascu-
lar medicine at the turn of the millennium: tri-
umphs, concerns, and opportunities. N Engl J Med.
1997;337(19):1360-1369.
2. Roger VL, et al. Heart disease and stroke statistics
- 2012 update: a report from the American Heart
Association. Circulation. 2012;125(1):e2-e220.
3. Lee RC, Feinbaum RL, Ambros V. The C. elegans
heterochronic gene lin-4 encodes small RNAs with
antisense complementarity to lin-14. Cell. 1993 ;
75(5):843-854.
4. Wightman B, Ha I, Ruvkun G. Posttranscriptional regulation of the heterochronic gene lin- 14 by lin- 4 mediates temporal pattern formation in C. elegans. Cell. 1993;75(5):855-862.

5. Krützfeldt J, et al. Silencing of microRNAs in vivo with 'antagomirs'. Nature. 2005;438(7068):685-689.

6. Kwon C, Han Z, Olson EN, Srivastava D. MicroRNA1 influences cardiac differentiation in Drosophila and regulates Notch signaling. Proc Natl Acad SciU S A. 2005;102(52):18986-18991.

7. Quiat D, Olson EN. MicroRNAs in cardiovascular disease: from pathogenesis to prevention and treatment. J Clin Invest. 2013;123(1):11-18.
8. Brigden W. Uncommon myocardial diseases. The non-coronary cardiomyopathies. Lancet. 1957; 2(7007):1179-1184.

9. Fuster V, Gersh BJ, Giuliani ER, Tajik AK, Brandenburg RP, Frye RL. The natural history of idiopathic dilated cardiomyopathy. Am J Cardiol. 1981;47(3):525-531.

10. Mestroni L, et al. Guidelines for the study of familial dilated cardiomyopathies. Collaborative Research Group of the European Human and Capital Mobility Project on Familial Dilated Cardiomyopathy. Eur Heart J. 1999;20(2):93-102. 
11. Herman DS, et al. Truncations of titin causing dilated cardiomyopathy. $N$ Engl J Med. 2012; 366(7):619-628.

12. McNally EM, Golbus JR, Puckelwartz MJ. Genetic mutations and mechanisms in dilated cardiomyopathy. J Clin Invest. 2013;123(1):19-26.

13. Andersson J, Libby P, Hansson G. Adaptive immunity and atherosclerosis. Clin Immunol. 2010; 134(1):33-46.

14. Ridker PM, Thuren T, Zalewski A, Libby P. Interleukin-1 $\beta$ inhibition and the prevention of recurrent cardiovascular events: rationale and design of the Canakinumab Anti-inflammatory Thrombosis Outcomes Study (CANTOS). Am Heart J. 2011:162(4):597-605.

15. Brigham and Women's Hospital. Cardioavscular Inflammation Reduction Trial (CIRT). NIH website. http://clinicaltrials.gov/ct2/show/NCT01594333? term $=$ low + dose + methotrexate\&rank $=3$. Updated June 22, 2012. Accessed November 5, 2012.

16. Lichtman AH, Binder CJ, Tsimikas S, Witztum JL. Adaptive immunity in atherogenesis: new insights and therapeutic approaches. J Clin Invest. 2013;123(1):27-36

17. van Berlo JH, Maillet M, Molkentin JD. Signaling effectors underlying pathologic growth and remodeling of the heart. J Clin Invest. 2013; 123(1):37-45.

18. Belin RJ, et al. Augmented protein kinase C- $\alpha$ induced myofilament protein phosphorylation contributed to myofilament dysfunction in experimental congestive heart failure. Circ Res. 2007; 101(2):195-204.

19. Liu Q, et al. Protein kinase C $\alpha$ but not PKC $\beta$ or PKC- $\gamma$, regulates contractility and heart failure susceptibility:implications of ruboxistaurin as a novel therapeutic approach. Circ Res. 2009; 105(2):194-200.

20. Malik FI, et al. Cardiac myosin activation: a potential therapeutic approach for systemic heart failure. Science. 2011;331(6023):1439-1443

21. Cleland JGF, et al. The effects of the cardiac myosin activator, omecamtiv mecarbil, on cardiac function in systolic heart failure: a double-blind, placebocontrolled, crossover, dose-ranging phase 2 trial. Lancet. 2011;378(9792):676-683.

22. Amgen. Study to Evaluate the Safety and Efficacy of IV Infusion Treatment with Omecamtiv Mecarbil in Subjects with Left Ventricular Systolic Dysfunction Hospitalized for Acute Heart Failure (ATOMIC-HF). NIH website. http://clinicaltrials. gov/ct2/show/NCT01300013?term=NCT013000 13\&rank=1. Updated October 10, 2012. Accessed November 5, 2012

23. Marks AR. Calcium cycling proteins and heart failure: mechanisms and therapeutics. J Clin Invest. 2013;123(1):46-52.

24. Gwathmey JK, et al. Abnormal intracellular calcium handling in myocardium from patients with end-stage heart failure. Circ Res. 1987;61(1):70-76.

25 . Bers DM. Ryanodine receptor S2808 phosphorylation in heart failure: smoking gun or red herring. Circ Res. 2012;110(6):796-799.

26. Hajjar RJ. Potential of gene therapy as a treatment for heart failure. J Clin Invest. 2013;123(1):53-61.

27. Wang J, Faust SM, Rabinowitz JE. The next step in gene delivery: molecular engineering of adenoassociated virus serotypes. J Mol Cell Cardiol. 2011; 50(5):793-802.

28. Miyamoto MI, et al. Adenoviral gene transfer of
SERCA2a improves left ventricular function in aortic-banded rats in transition to heart failure. Proc Natl Acad Sci U S A. 2000;97(2):793-798.

29. Lyon AR, et al. SERCA2a gene transfer decreases sarcoplasmic reticulum calcium leak and reduces ventricular arrhythmias in a model of chronic heart failure. Circ Arrbythm Electrophysiol. 2011;4(3):362-373.

30. Mariani JA, Smolic A, Preovolos A, Byrne MJ, Power JM, Jaye DM. Augmentation of left ventricular mechanics by recirculation-mediated AAV2/1-SERCA2a gene delivery in experimental heart failure. Eur J Heart Fail. 2011;13(3):247-253.

31. Jessup $M$, et al. Calcium upregulation by percutaneous administration of gene therapy in cardiac disease (CUPID): a phase 2 trial of intracoronary gene therapy of sarcoplasmic reticulum $\mathrm{Ca} 2+$ ATPase in patients with advanced heart failure. Circulation. 2011;124(3):304-313.

32. Karsner HT, Saphir O, Rodd TW. The state of the cardiac muscle in hypertrophy and atrophy. Am J Pathol. 1925;1(4):351-371.

33. Linzbach AJ. Heart failure from the point of view of quantitative anatomy. Am J Cardiol. 1960:5:370-382.

34. Beltrami AP, et al. Evidence that human cardiac myocytes divide after myocardial infarction. NEngl JMed. 2001;344(23):1750-1757.

35. Kajstura J, Leri A, Finato N, DiLoreto C, Beltrami CA, Anversa P. Myocyte proliferation in end-stage cardiac failure in humans. Proc Natl Acad Sci U S A. 1998;95(15):8801-8805

36. Olivetti $G$, et al. Apoptosis in the failing human heart. N Engl J Med. 1997;336(16):1131-1141.

37. Bergmann O, et al. Evidence for cardiomyocyte renewal in humans. Science. 2009;324(5923):98-102.

38. Bearzi C, et al. Human cardiac stem cells. Proc Natl Acad Sci U S A. 2007;104(35):14068-14073.

39. Anversa P, Kajstura J, Rota M, Leri A. Regenerating new heart with stem cells. J Clin Invest. 2013;123(1):62-70.

40. Degenhardt K, Singh MK, Epstein JA. New approaches under development: cardiovascular embryology applied to heart disease. J Clin Invest. 2013;123(1):71-74

41. Epstein JA. Cardiac development and implications for heart disease. $N$ Engl J Med. 2010; 363(17):1638-1647.

42. Singh MK, Epstein JA. Epicardium-derived cardiac mesenchymal stem cells expanding the outer limit of heart repair. Circ Res. 2012;110(7):904-906.

43. Smart N, et al. De novo cardiomyocytes from within the activated adult heart after injury. Nature. 2011;474(7353):640-644

44. Porrello ER, et al. Transient regenerative potential of the neonatal mouse heart. Science. 2011; 331(6020):1078-1080.

45. George AL Jr. Molecular and genetic basis of sudden cardiac death. J Clin Invest. 2013;123(1):75-83

46. Cerrone M, Napolitano C, Priori SG. Genetics of ion-channel disorders. Curr Opin Cardiol. 2012;27(3):242-252.

47. Ackerman MJ, et al. HRS/EHRA Expert Consensus Statement on the state of genetic testing for the channelopathies and cardiomyopathies. Heart Rhythm. 2011;8(8):1308-1339.

48. Priori SG, Napolitano C, Di Pasquale E, Condorelli G. Induced pluripotent stem cell-derived cardiomyocytes in studies of inherited arrhythmias. J Clin Invest. 2013;123(1):84-91.

49. Takahashi K, Yamanaka S. Induction of pluripotent stem cells from mouse embryonic and adult fibroblast cultures by defined factors. Cell. 2006;126(4):663-676

50. Davis RP, van den Berg CW, Casini S, Braam SR, Mummery CL. Pluripotent stem cell models of cardiac disease and their implication for drug discovery and development. Trends Mol Med. 2011; 17(9):475-484.

51. Itzhaki I, et al. Modelling the long QT syndrome with induced pluripotent stem cells. Nature. 2011; 471(7337):225-229.

52. Yazawa M, et al. Using induced pluripotent stem cells to investigate cardiac phenotypes in Timothy syndrome. Nature. 2011;471(7337):230-234.

53. Obraztov VP, Strazhesko ND. Simtomatiologii I diagnostike tromboza venecchinikh arterial cerdtsa. In: Vorobeva VA, Konchalovski MP, eds. Trudi Pervogo Sesda Rossushkikh Terapevtov. Vol 10. Moscow, Russia: Tovareschejestvo Tepografe, A.E. Mamontov; 1910:26-43 (Russian).

54. Braunwald E. The treatment of acute myocardial infarction: the past, the present, and the future. Eur Heart J Acute Cardiovasc Care. 2012;1(1):9-12.

55. Julian DG. Treatment of cardiac arrest in acute myocardial ischemia and infarction. Lancet. 1961; 2(7207):840-844.

56. Maroko PR, et al. Factors influencing infarct size following experimental coronary artery occlusion. Circulation. 1971;43(1):67-82.

57. Chazov EI, Matveeva LS, Mazaev AV, Sargin KE, Sadovskaia GV, Ruda MI. Intracoronary administration of fibrinolysin in acute myocardial infarction. Ter Arkh. 1976;48(4):8-19.

58. Braunwald E, Kloner RA. Myocardial reperfusion: a double-edged sword? J Clin Invest. 1985; 76(5):1713-1719.

59. Hausenloy DJ, Yellon DM. Myocardial ischemiareperfusion injury: a neglected therapeutic target. J Clin Invest. 2013;123(1):92-100.

60. Murry CE, Jennings RB, Reimer KA. Preconditioning with ischemia: a delay of lethal cell injury in ischemic myocardium. Circulation. 1986;74(5):1124-1136.

61. Przyklenk K, Bauer B, Ovize M, Kloner RA, Whittaker P. Regional ischemic "preconditioning" protects remote virgin myocardium from subsequent sustained coronary occlusion. Circulation. 1993; 87(3):893-899.

62. Zhao ZQ, et al. Inhibition of myocardial injury by ischemic postconditioning during reperfusion: comparison with ischemic preconditioning. Am J Physiol Heart Circ Physiol. 2003;285(2):H579-H588.

63. Braunwald E. Clinical efforts to reduce myocardial infarct size - the next step.J Cardiovasc Pharm Ther. 2011;16(3-4):349-353.

64. Furchgott RF, Zawadzki JV. The obligatory role of endothelial cells in the relaxation of arterial smooth muscle by acetylcholine. Nature. 1980; 288(5789):373-376

65 . Koshland DE Jr. The molecule of the year. Science. 1992;258(5090):1861.

66. Stamler JS, et al. S-nitrosylation of proteins with nitric oxide: synthesis and characterization of biologically active compounds. Proc Natl Acad Sci US A. 1992;89(1):444-448.

67. McMahon TJ, et al. A nitric oxide processing defect of red blood cells created by hypoxia: deficiency of S-nitrosohemoglobin in pulmonary hypertension. Proc Natl Acad Sci U S A. 2005;102(41):14801-14806. 68. Haldar SM, Stamler JS. S-nitrosylation: integrator of cardiovascular performance and oxygen delivery. J Clin Invest. 2013;123(1):101-110. 